\title{
HOMOGENIZATION OF A CONDUCTIVE AND RADIATIVE HEAT TRANSFER PROBLEM, SIMULATION WITH CAST3M
}

\author{
K. El Ganaoui ${ }^{1}$ \\ SFME/LTMF, bat 454 \\ CEA Saclay 91191 Gif-sur-Yvette \\ France \\ Email: kelganaoui@cea.fr
}

\author{
G. Allaire \\ CMAP, Ecole Polytechnique \\ 91128 Palaiseau \\ France \\ Email: allaire@cmapx.polytechnique.fr
}

\begin{abstract}
We are interested in conductive and radiative transfer of energy in the core of gas cooled reactors. Two scales characterize the problem: macroscopic and microscopic. We want to consider the domain like an equivalent homogenous medium. So we use homogenization theory to compute the effective macroscopic properties which take into account the microscopic structure. We first present a full mathematical study of a simpler conduction problem with non linear boundary condition and its simulation with the CEA's (French Atomic Energy Commissariat) computer code CAST3M. Then we present the homogenization of the real physical problem (including radiative boundary condition).
\end{abstract}

\section{NOMENCLATURE}

$d$ dimension of the space.

$\sigma$ Stefan-Boltzmann constant.

$K$ conductivity matrix.

$K^{*}$ homogenized conductivity matrix.

$\Omega_{\varepsilon} \quad$ perforated periodic domain.

$\Omega$ non perforated domain.

$\varepsilon$ period of the domain $=\frac{\text { size of the cell }}{\text { size of the domain }}$.

$\Gamma_{\varepsilon, i}$ boundary of a given channel $i$ in $\Omega_{\varepsilon}$.

$x$ macroscopic variable.

$y=\frac{x}{\varepsilon} \quad$ microscopic variable.

$T_{\varepsilon} \quad$ temperature, solution of the real problem.

$T$ temperature, solution of the homogenized problem.

$f$ volumetric source.

$g$ Neumann imposed flux.

Id identity operator.

\footnotetext{
${ }^{1}$ Address all correspondence to this author.
}

\section{INTRODUCTION}

The Gas Cooled Reactor $(G C R)$ core contains a large number of hexagonal blocks. Each block is made of a solid moderator perforated periodically by many cylindrical gas channels (see Figure 1, Figure 2). In our study, the heat exchange is done by conduction in the solid and by radiation on the walls of the channels (gas considered like vacuum: no heat conduction and no absorption of radiation). The diameter of channels is very small compared to the global dimension. So two scales characterize the domain (macroscopic and microscopic). The corresponding mathematical model has therefore highly heterogeneous coefficients that make the problem numerically very difficult to treat (memory and CPU time). We seek to consider the core like an equivalent homogenous medium, a mixture of gas and solid. Homogenization theory (see Bensoussan) has been used to compute the effective macroscopic properties which take into account the microscopic structure. The resulting homogenized model is simpler to simulate: first compute so-called cell problems to obtain the values of the homogenized coefficients, second solve a simpler macroscopic effective equation with a coarse mesh. The process of homogenization that we present here allows us to retrieve the average value of the temperature and allows also to obtain a correcting term which gives a far more accurate result. The simulation is done using CAST3M which is a product of the French Atomic Energy Commissariat(Agency). It's a general purpose code for solving partial differentials equations by the finite element method. Application domains are structural mechanics, fluid mechanics, heat transfer thermodynamics, magnetism. 
In our application, the walls of channels are diffuse emitters and reflectors. In thermal engineering applications, surfaces are gray and their emissivity is between 0 and 1 . To simplify the problem we choose the case of emissivity equal to 1 .

For our study, a periodic perforated domain $\Omega_{\varepsilon}$, representative of the real geometry has been considered. $\Omega_{\varepsilon}$ is obtained from an initial solid domain $\Omega$ with $\partial \Omega$ as boundary. we denote by $\varepsilon$ the domain period (positive and very small compared to the global size). $Y$ is the reference periodicity cell (see Figure 3) and $\Gamma$ is the boundary of the perforations contained in $Y . Y^{*}$ is the solid part of $Y$.

The aim now is to find $T_{\varepsilon}$ solution of the following system:

$\left\{\begin{array}{l}-\operatorname{div}\left(K \nabla T_{\varepsilon}\right)=f \quad \text { in } \quad \Omega_{\varepsilon} \\ K \nabla T_{\varepsilon} \cdot n=g \quad \text { on } \partial \Omega \\ -K \nabla T_{\varepsilon} \cdot n=\frac{1}{\varepsilon}\left[\sigma T_{\varepsilon}^{4}-\int_{\Gamma_{\varepsilon, i}} \sigma w(x, z) T_{\varepsilon}^{4}(z) d z\right] \text { on } \Gamma_{\varepsilon, i} .\end{array}\right.$

$w(x, z)$ is the view factor between two given points $x$ and $z$ of the radiative surface $\Gamma_{\varepsilon, i}$. we define also for a given function $\varphi$ an operator $k$ (see Tiihonen) for the mathematical properties of this operator) by:

$$
k(\varphi)(x)=\int_{\Gamma_{\varepsilon, i}} w(x, z) \varphi(z) d z \quad \text { for } \quad s \in \Gamma_{\varepsilon, i}
$$

The radiative boundary condition (third equation of (1)) can also be written as:

$$
-K \nabla T_{\varepsilon} \cdot n=\frac{1}{\varepsilon}(I d-k)\left(\sigma T_{\varepsilon}^{4}\right) \quad \text { on } \quad \Gamma_{\varepsilon, i}
$$

The domain $\Omega_{\varepsilon}$, with its conductivity $K\left(\frac{x}{\varepsilon}\right)$, is highly heterogenous with periodic heterogeneities of length-scale $\varepsilon$. From a numerical point of view, solving the problem (1) by any method will require too much effort if the period $\varepsilon$ is small. It's preferable to average or homogenize the properties of $\Omega_{\varepsilon}$ and compute an approximation of $T_{\varepsilon}$ on a coarse mesh. Our goal is to study the behavior of $T_{\varepsilon}$ when $\varepsilon$ tends to 0 . In other words, we will approximate the temperature for a very large number of assemblies in the core.

\section{HOMOGENIZATION OF A SIMPLER PROBLEM}

First of all we homogenized a simpler conduction problem with a non linear boundary condition that can be interpreted as a radiative heat exchange with an infinite medium characterized by a constant temperature $T_{\infty}$. In this case The problem to solve is to find $T_{\varepsilon}$ solution of

$$
\left\{\begin{array}{l}
-\operatorname{div}\left(K \nabla T_{\varepsilon}\right)=f \quad \text { in } \quad \Omega_{\varepsilon} \\
K \nabla T_{\varepsilon} \cdot n \quad=g \quad \text { on } \quad \partial \Omega \\
-K \nabla T_{\varepsilon} \cdot n=\varepsilon \sigma\left(T_{\varepsilon}^{4}-T_{\infty}^{4}\right) \quad \text { on } \quad \Gamma_{\varepsilon, i}
\end{array}\right.
$$

To homogenize the problem we start from the following two-scale asymptotic expansion of the temperature:

$$
T_{\varepsilon}(x)=T\left(x, \frac{x}{\varepsilon}\right)+\varepsilon T_{1}\left(x, \frac{x}{\varepsilon}\right)+\varepsilon^{2} T_{2}\left(x, \frac{x}{\varepsilon}\right)+\ldots
$$

where $T_{j}\left(x, \frac{x}{\varepsilon}\right)$ are $Y$-periodic functions defined in $\Omega \times Y$. Substituting in (4) $T_{\varepsilon}$ by the relationship (5) we obtain a cascade of problems. From this problems we can show that the first term of (5) depends only of the macroscopic variable $x$ and that:

$$
T_{1}(x, y)=\sum_{i=1}^{d} \frac{\partial T}{\partial x_{i}}(x) \omega_{i}(y)
$$

where $\omega_{i}, i=1, \ldots, d$ are the solutions of the cell problems given by:

$$
\left\{\begin{array}{l}
-\operatorname{div}_{y}\left(K\left(e_{i}+\nabla \omega_{i}\right)\right)=0 \quad \text { in } \quad Y^{*} \\
-K\left(e_{i}+\nabla w_{i}\right) \cdot n=0 \quad \text { on } \Gamma \\
y \longmapsto \omega_{i}(y) \quad \text { Y-periodic }
\end{array}\right.
$$

where $e_{i}$ are the vectors of the canonical basis.

The homogenized problem that models the mean behavior of the problem (4) is then given by: find the temperature $T$ as solution of the following system

$$
\left\{\begin{array}{l}
-\operatorname{div}\left(K^{\star} \nabla T\right)+\sigma \alpha_{1}\left(T^{4}-T_{\infty}^{4}\right)=\alpha_{2} f \quad \text { in } \quad \Omega \\
-K^{\star} \nabla T \cdot n=\operatorname{mes}(Y) g \text { on } \partial \Omega
\end{array}\right.
$$

where $K^{\star}$ is the homogenized conductivity matrix. Its coefficients use the solutions of cell-problems:

$$
K_{i j}^{*}=\int_{Y^{*}} K\left(e_{i}+\nabla w_{i}\right) \cdot\left(e_{j}+\nabla w_{j}\right) d y
$$

$\alpha_{i}$ are homogenized terms which depend on the geometry of the cell.

A full mathematical study of this problem has been done, we have showed the two-scale convergence (see Allaire) of the problem (4) toward its homogenization: the problem (8). The results of simulation with the computer code CAST3M allowed to find the theoretical rate of convergence (see Figure 9). 


\section{HOMOGENIZATION OF THE CONDUCTION-RADIATION PROBLEM}

The real physical problem including the radiative boundary condition (1), introduced in the beginning, has also been studied through a two-scale asymptotic expansions. The second term of the expansion is still given by (6) where the $\omega_{i}$, are now solutions of the following cell problems:

$$
\left\{\begin{array}{l}
-\operatorname{div}_{y}\left(K\left(e_{i}+\nabla \omega_{i}\right)\right)=0 \quad \text { in } Y^{*} \\
-K\left(e_{i}+\nabla \omega_{i}\right) \cdot n=4 \sigma T^{3}(I-k)\left(\omega_{i}+y_{i}\right) \quad \text { on } \quad \Gamma \\
y \longmapsto \omega_{i}(y) \quad \text { Y-periodic }
\end{array}\right.
$$

The homogenized problem is:

$$
\left\{\begin{array}{l}
\left.-\operatorname{div}_{y}\left(K^{*}\left(T^{3}\right) \nabla T\right)\right)=\beta_{1} f \text { in } \quad \Omega \\
-K^{*}\left(T^{3}\right) \nabla T \cdot n=\beta_{2} g \text { on }-\partial \Omega
\end{array}\right.
$$

Where $\beta_{i}$ are homogenized terms which depend on the geometry of the cell. The coefficients $K_{i, j}^{*}$ are given now as follow:

$K_{i j}^{*}=\int_{Y^{*}} K\left(\nabla \omega_{i}+e_{i}\right) \cdot\left(\nabla \omega_{j}+e_{j}\right)+4 \sigma T^{3} \int_{\Gamma}(I-k)\left(\omega_{i}+y_{i}\right)\left(\omega_{j}+y_{j}\right)$

Note that the coefficients depend as well of the cell problems and of the macroscopic temperature. It's a consequence of the fact that they take into account both conductive and radiative heat transfer.

\section{SIMULATIONS}

The results of simulation with CAST3M are given in the case of the problem (4) in $2 D$, following the algorithm bellow

\section{Resolution algorithm}

1. First solve in the cell geometry (see Figure 3) the cell problems (one problem in each direction of the space). Solutions will allow us to compute the homogenized conductivity matrix coefficients $K_{i j}^{*}(9)$.

2. Second solve the homogenized problem that provides the homogenized temperature $T(8)$.

3. Third, $T$ and $\omega_{i}$ allow us to calculate the term of correction $\varepsilon T_{1}\left(x, \frac{x}{\varepsilon}\right)$ to enhance the approximation of $T_{\varepsilon}$.

\section{Results of simulation with CAST3M}

In the Figure 4 we see the solutions of the cell problems (7). Figure 5 is the solution of the homogenized problem resolved in $\Omega$ (non perforated domain) Figure 6 is result of the homogenization process. Figure 7 gives the results of the direct resolution of the non-homogenized problem (i.e with a mesh of the perforated domain $\Omega_{\varepsilon}$ ). Figure 9 shows the convergence of the relative error on the temperature when the period $\varepsilon \rightarrow 0$ compared to the curve of theoretical rate of convergence.

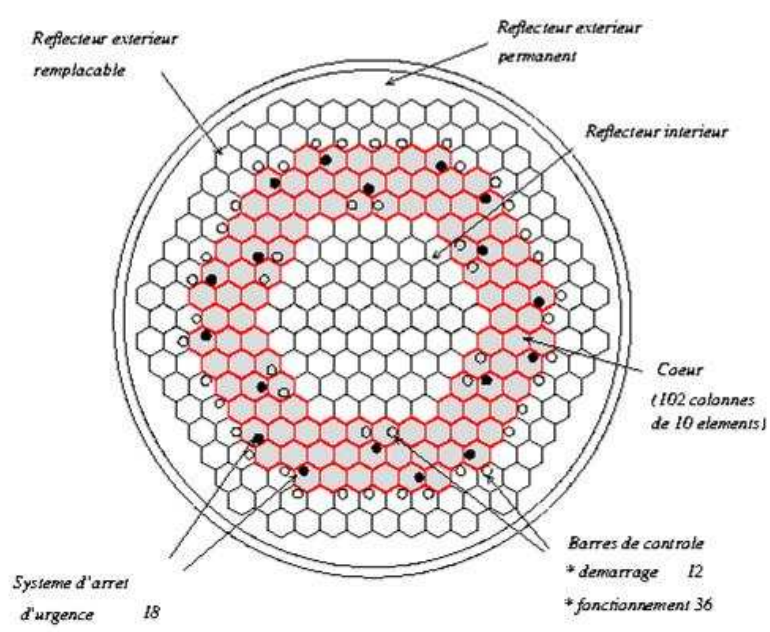

Figure 1. $2 D$ section of the reactor core each red cell contains 10 of the cell bellow

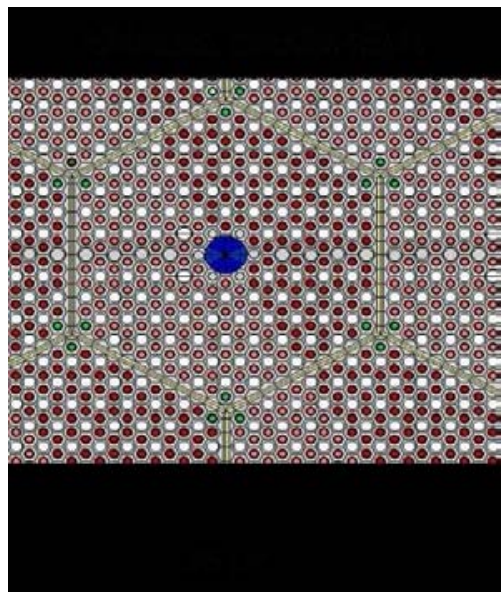

Figure 2. one fuel cell of the core 


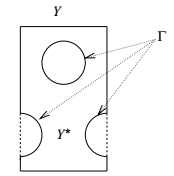

Figure 3. The chosen cell model

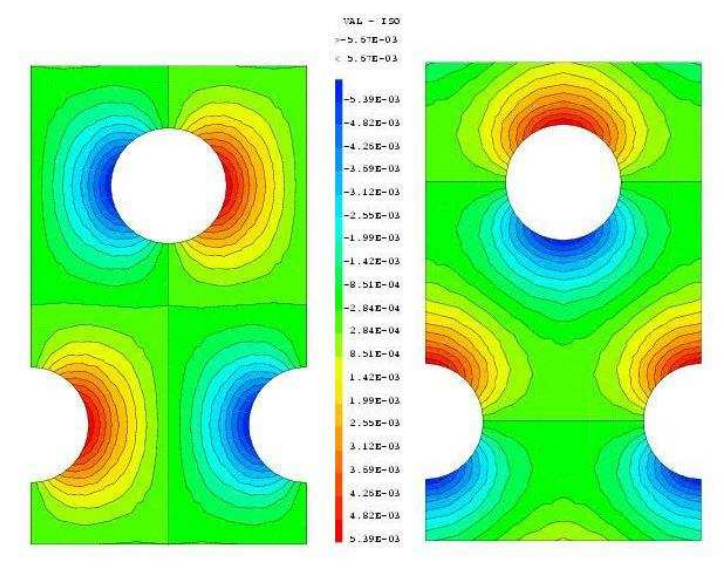

GRE FECTI

Figure 4. $\omega_{1}\left(\frac{x}{\varepsilon}\right)$ and $\omega_{2}\left(\frac{x}{\varepsilon}\right)$ : solutions of cell problems

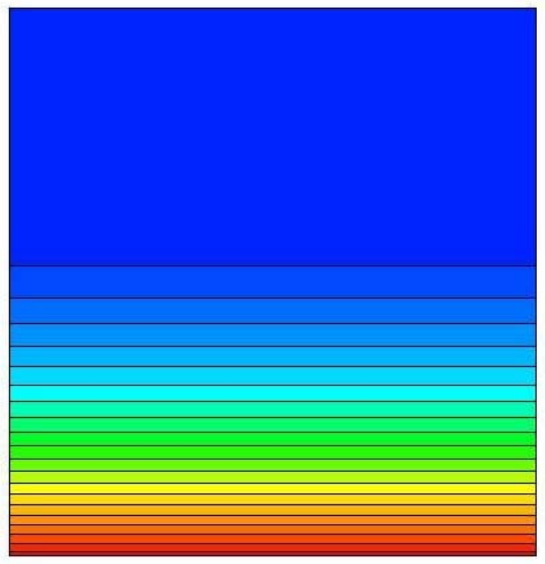

Figure 5. $T$, solution of the homogenized problem

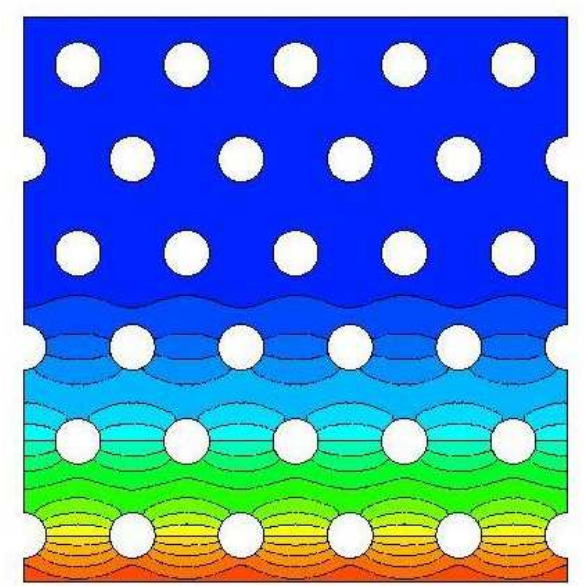

Figure 6. $T(x)+\varepsilon T_{1}\left(x, \frac{x}{\varepsilon}\right)$ : Reconstructed temperature

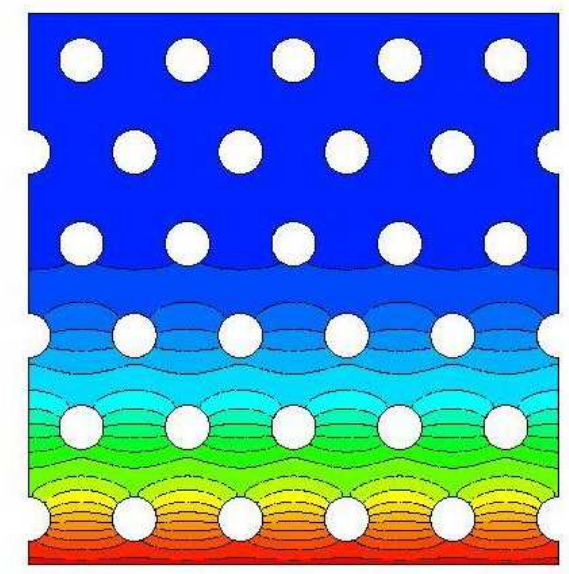

Figure 7. $T_{\varepsilon}(x)$ : Direct computation 
VAL - I 0

> 5. $60 x+02$

< $6 . T 3 x+a z$

5. T3E+ OZ

5. TBL+ 0Z

5. $83 E+0 z$

5. $832+02$

5. $93 \boldsymbol{x}+0 \mathrm{z}$

5. $98 x+0 z$

$6.032+0 z$

6. $08 x+02$

6. $13 E+0 z$

6. $18 E+0 z$

6. $23 x+0 z$

6. $28 x+0 z$

6. $33 x+02$

6. $38 x+02$

6. $43 E+0 Z$

6. $48 \mathrm{E}+0 \mathrm{Z}$

$6.53 x+0 z$

$6.58 x+02$

6. $63 x+0 z$

$6.68 \mathrm{x}+0 \mathrm{z}$

6. T3X+ 0Z

Figure 8. The colorbar for temperatures $T, T_{\varepsilon}$ and $T(x)+\varepsilon T_{1}\left(x, \frac{x}{\varepsilon}\right)$

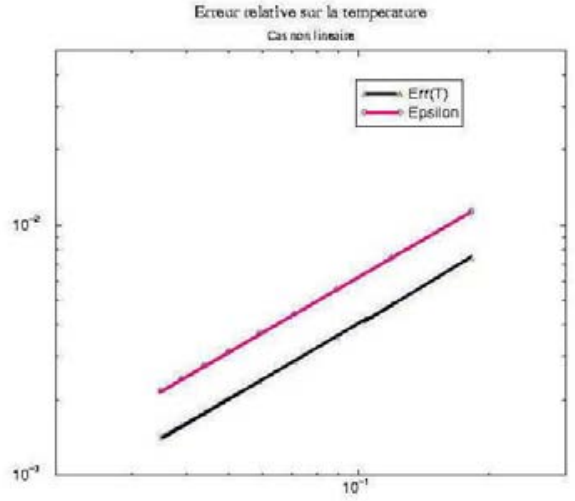

Figure 9. curve of the convergence of the error: $\left\|T_{\varepsilon}-\left(T+\varepsilon T_{1}\right)\right\|_{L^{2}}$ in function of $\varepsilon$ compared to $\varepsilon$

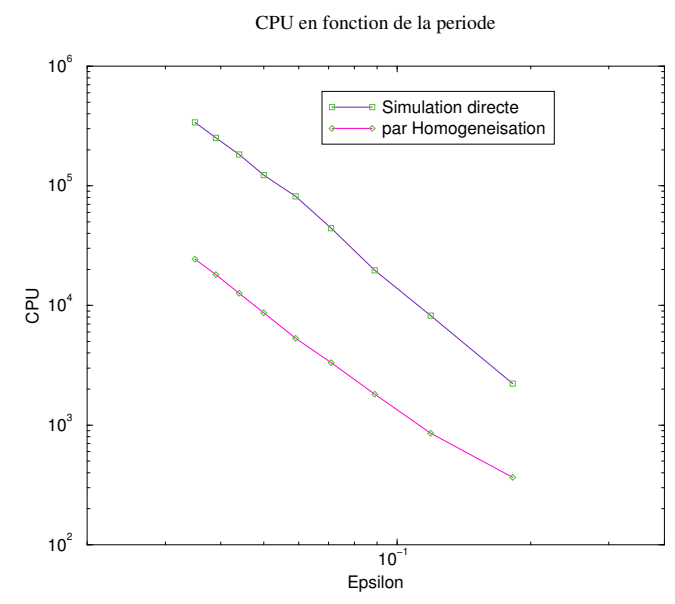

Figure 10. CPU time compared between direct resolution and with homogenization 


\section{CONCLUSION}

This work was an important preliminary step in the study of the problem including the non linear but also non local boundary condition on the radiating part of the boundary (i.e. on the wall of each gas channel). It allowed to prove the feasibility of the homogenization method implemented in CAST3M code. One of the main result of this stage is the saving of CPU time compared to the direct computation. The simulation of the complete problem (1) is under progress. The next step will be to consider an emissivity value between 1 and 0 and may be a coupling between thermic model and fluid mechanics equations.

\section{REFERENCES}

Allaire, G., Homogenization and Two-Scale Convergence, SIAM J. Math. Anal., Vol.23, No. 6, pp. 1482-1518, November 1992.

Bensoussan, A., Lions, J.L. and Papanicolaou, G., Asymptotic Analysis for Periodic Structures, NorthHolland, Amsterdam, 1978.

http://www-cast3m.cea.fr/cast3m/index.jsp.

Modest, M., Radiative Heat Transfer, Mc Graw-Hill, New York, 1993.

Tiihonen, T., Stefan -Boltzmann Radiation on Nonconvex Surfaces, Mathematical Methods in the Applied Sciences, Vol. 20, 47-57, 1997. 